\title{
Nanostructured surfaces: cell engineering and
} cell biology

"This is a fast moving field involving cutting edge material developments and stem cell biology. Forums such as Nanomedicine will help to bridge disciplines and allow us to generate next-generation materials for cell and tissue engineering."

Recently, Hench and Polak described thirdgeneration biomaterials as materials that will influence cells at the molecular level [1]. This is an intriguing thought. Clearly, all material interactions will alter cells at a molecular level, however, the idea of altering specific transcription factors and genes turning off and on specific cell differentiation/action pathways is very exciting. In this editorial, I would like to consider next-generation material influence of mesenchymal stem cells (MSCs) as a cell type that could potentially underpin cell and tissue engineering.

We must, first, however, work out how this can be done. There presently seems to be three main routes of controlling MSC differentiation using materials: chemistry, stiffness and topography. McBeath et al. demonstrated that through cell confinement strategies using microcontact printing of fibronectin, alterations in cell shape either adipogenic or osteogenic pathways could be stimulated [2]. Engler et al. showed that MSC differentiation could be influenced through changes in modulus from very soft $(0.1-1 \mathrm{kPa}$ : neurogenic) to less soft (8-17 $\mathrm{kPa}$ : myogenic) to quite hard (25-40 kPa: osteogenic) [3]. Following this, we showed that nanoscale changes in topography could alter MSC differentiation [4]. We did this using electron beam lithography (EBL) to generate nanoscale patterns ranging from highly ordered (the typical approach using EBL) through controlled disorder to total randomness (the typical approach using, for example, etching, anodization or blasting) and demonstrated that successful osteoconversion of MSCs was achieved using a $\pm 50 \mathrm{~nm}$ level of disorder. This demonstrates a unique middle route from the traditional (ordered or random) approaches. All three routes can be said to be influencing the MSCs on the nanoscale level and can also be considered to be biomimetic: constrain cells to the shape you want and they form the tissue you want; grow the cells on the stiffness of tissue you desire and you will get that tissue; add in biomimetic levels of disorder (i.e., biological standard deviation) and the cells will be triggered appropriately.

It seems clear that these biomimetic cues are probably working through influencing cells at the molecular level, but how is a key question - and one that urgently needs to be answered if we are to generate third-generation biomaterials. To achieve this, there needs to be a nexus between the biological and physical sciences with excellence in cell biology connecting to the latest in nanoscale engineering, physics and chemistry.

The answer to these questions may lie in the cells' focal adhesions. These are key structures that interact with the material surface and are central to many signaling cascades. Such signaling pathways include G-protein and cytoskeletal signaling (e.g., Rho, Rac and Cdc 42), which modulate cell 'sensing', shape and contractility, and MAPKs, for example ERK and p38 MAPK, which are downstream effectors of a wide range of transcription factors involved in cell survival, proliferation and differentiation. In my mind, it is these transcription factors that are key to control through material-mediated modulation of focal adhesions.

Another, highly speculative, way of modulating transcription factor activity may be through more direct cytoskeletal signaling [5] effected by focal adhesion-mediated distortion of the nucleus. Chemical, topographical and Young's modulus changes will influence adhesion size, shape and number [6] and hence change cytoskeletal arrangements. Ingber's elegant theory of cellular tensegrity $[7,8]$ shows us how the nucleus may be connected to the cytoskeleton. The nucleus itself possesses a lamin nucleoskeleton that is closely associated with interphase chromosomes. Thus, by aligning theories by Ingber and Forgacs [9], the cells can be said to be like a spider in a web - the web being the extracellular environment and mechanical changes

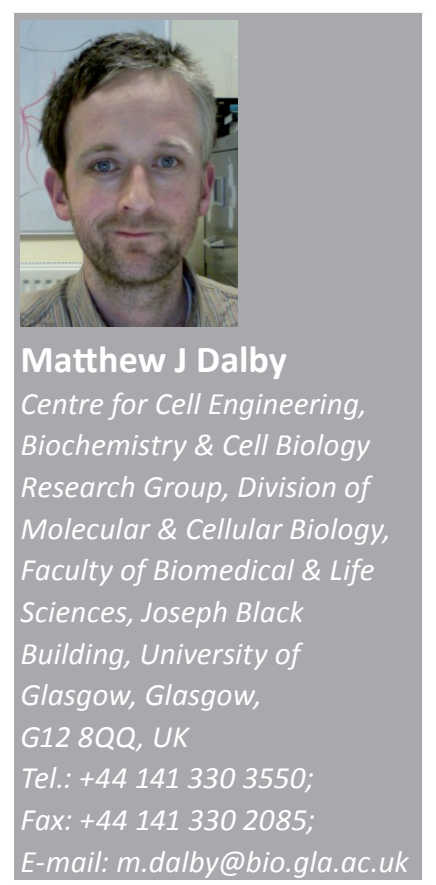

"Through understanding the cell biology of cells cultured on materials in more detail, we will be able to develop third-generation nanoscale biomaterials. Thus ... there needs to be far more emphasis on the basic biological sciences."

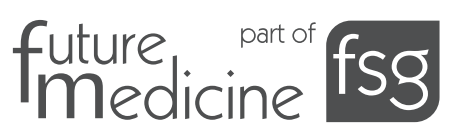


(e.g., changes in the cells' ability to spread) being transmitted through the cytoskeleton (the spiders legs) to the nucleus (the spiders body); thus describing a percolation network [9]. Passing these mechanical changes to the nucleus may change the location of key genes to transcription factors, thus altering genomic expression patterns and cell phenotype [10].

Through understanding the cell biology of cells cultured on materials in more detail, we will be able to develop third-generation nanoscale biomaterials. Thus, it is my opinion that there needs to be far more emphasis on the basic biological sciences in order to enable the applied and clinical sciences.

In terms of materials, there are now two main goals, again fitting into basic and applied sciences. First, we need to strive for novel surfaces to support cell growth in defined ways - for example, switchable surfaces [11] and self-organizing surfaces $[12,13]$. The other big push has to be to develop 3D scaffolds with these cell cues in-built (recently reviewed in [14])

This is a fast moving field involving cutting edge material developments and stem cell biology. Forums such as Nanomedicine will help to bridge disciplines and allow us to generate next-generation materials for cell and tissue engineering.

\section{Financial \& competing interests disclosure}

The author has no relevant affiliations or financial involvement with any organization or entity with a financial interest in or financial conflict with the subject matter or materials discussed in the manuscript. This includes employment, consultancies, honoraria, stock ownership or options, expert testimony, grants or patents received or pending, or royalties.

No writing assistance was utilized in the production of this manuscript.

\section{Bibliography}

1 Hench LL, Polak JM: Third-generation biomedical materials. Science 295, 1014-1017 (2002).

2 McBeath R, Pirone DM, Nelson CM, Bhadriraju K, Chen CS: Cell shape, cytoskeletal tension, and RhoA regulate stem cell lineage commitment. Dev. Cell 6, 483-495 (2004).

3 Engler AJ, Sen S, Sweeney HL, Discher DE: Matrix elasticity directs stem cell lineage specification. Cell 126, 677-689 (2006).

4 Dalby MJ, Gadegaard N, Tare R et al.: The control of human mesenchymal cell differentiation using nanoscale symmetry and disorder. Nat. Mater. 6, 997-1003 (2007).

5 Curtis ASG, Dalby MJ, Gadegaard N: Cell signaling arising from nanotopography: implications for nanomedical devices. Nanomedicine 1, 67-72 (2006).
6 Biggs MJ, Richards RG, McFarlane S, Wilkinson CD, Oreffo RO, Dalby MJ: Adhesion formation of primary human osteoblasts and the functional response of mesenchymal stem cells to $330 \mathrm{~nm}$ deep microgrooves. J. R. Soc. Interface 5, 1231-1242 (2008)

7 Ingber DE: Tensegrity II. How structural networks influence cellular information processing networks. J. Cell Sci. 116, 1397-1408 (2003).

8 Ingber DE: Tensegrity I. Cell structure and hierarchical systems biology. J. Cell Sci. 116, 1157-1173 (2003).

9 Shafrir Y, Forgacs G: Mechanotransduction through the cytoskeleton. Am. J. Physiol. Cell. Physiol. 282, C479-C486 (2002).

10 Dalby MJ, Biggs MJ, Gadegaard N, Kalna G, Wilkinson CD, Curtis AS: Nanotopographical stimulation of mechanotransduction and changes in interphase centromere positioning. J. Cell. Biochem. 100, 326-338 (2007).

11 Todd SJ, Farrah D, Gough JE, Ulijn RV: Enzyme-triggered cell attachment to hydrogel surfaces. Soft Materials 3, 547-550 (2007).

12 Silva GA, Czeisler C, Niece KL et al.: Selective differentiation of neural progenitor cells by high-epitope density nanofibers. Science 303, 1352-1355 (2004).

13 Williams RJ, Smith AM, Collins R, Hodson N, Das AK, Ulijn RV: Enzyme-assisted self-assembly under thermodynamic control. Nat. Nanotechnol. 4, 19-24 (2009).

14 Seunarine K, Gadegaard N, Tormen M, Meredith DO, Riehle MO, Wilkinson CD: $3 \mathrm{D}$ polymer scaffolds for tissue engineering. Nanomedicine 1, 281-296 (2006). 